\title{
FOXM 1 Expression in Breast Carcinoma and Correlation with Histopathological Staging and Grading
}

\author{
Rafah Mohamed Jaffar Alkhateeb ${ }^{1}$, Salim Rasheed Alaubaidy ${ }^{2}$ \\ ${ }^{1}$ Imamain Kadhymien Teaching Hospital, Baghdad, Iraq \\ ${ }^{2}$ Pathology Department \& Forensic Medicine, College of Medicine/University of Baghdad, Baghdad, Iraq \\ Email address: \\ rafahmalkhateeb@gmail.com (R. M. J. Alkhateeb),dr_salimhamoudi@yahoo.com (S. R. Alaubaidy)
}

\section{To cite this article:}

Rafah Mohamed Jaffar Alkhateeb, Salim Rasheed Alaubaidy. FOXM 1 Expression in Breast Carcinoma and Correlation with Histopathological Staging and Grading. Pathology and Laboratory Medicine. Vol. 2, No. 1, 2018, pp. 15-19. doi: $10.11648 /$ j.plm.20180201.13

Received: March 13, 2018; Accepted: April 11, 2018; Published: May 17, 2018

\begin{abstract}
FOXM1 (Forkhead box protein M1) promoted EMT (Epithelial-to-mesenchymal transition) in breast cancer by binding and activation of the promoter of SLUG gene. that FOXM1 promotes breast cancer metastases by activation of the TGF- $\beta$ pathway with through interaction SMAD3 (this prevented E3 ubiquitin-protein ligase transcriptional intermediary factor $1 \gamma$ [TIF1 $\gamma$ ] binding to SMAD3 and protected SMAD4 from ubiquitination) that leads to stabilization of the SMAD3/SMAD4 complex. The study samples included seventy four formalin-fixed, paraffin embedded tissue blocks which have been diagnosed as forty four cases of breast carcinoma and their lymph nodes. In the present study from 74 total cases, 46 cases $(62.6 \%)$ were showing FOXM1 marker positive while 28 cases $(37.8 \%)$ were showing negative immunohistochemistry for FOXM1 marker. Sub classification of malignant cases, 16 malignant breast cancer cases (36.4\%) showed no FOXM1 expression, while 12 malignant cases $(27.3 \%)$ were strongly positive for FOXM1 expression. There is significant correlation between breast carcinoma grade, stage and FOXM1 expression.
\end{abstract}

Keywords: Breast Tumor, Breast Cancer, FOXM1 Expression

\section{Introduction}

Cancer is a major public health problem worldwide and is the second leading cause of death in the United States. Breast cancer (BC) starts when cells in the breast begin to grow out of control as the cancerous cells grow and multiply, they form a malignant tumor within the breast. [1] cancer stem cells (CSC) or tumor-initiating cells. [2] CSC possesses selfrenewal properties and can differentiate into daughter cells. However, they differ from stem cells since they are tumorigenic and thus have the ability to form tumors when transplanted into animals whereas stem cells do not have this ability. [3] CSC also has increased chemoresistance, can promote metastasis and can survive treatment resulting in new tumors causing relapses. [4] The first identification of a CSC in solid tumors came from the work of Al-Hajj et al. [3] using cells isolated from primary tumors of breast cancer patients. Cells with an ESA+, CD44+, CD24-. Forkhead box protein $\mathrm{M} 1$ is a protein that in humans is encoded by the FOXM1 gene [4] FoxM1, also known as Trident, MPP2, or
HFH-11, belongs to the large family of forkhead transcription factors. The protein encoded by this gene is a member of the FOX family of transcription factors [5] FOXM1 has been awarded the Molecule of the Year 2010 for its growing potential as a target for cancer diagnosis and therapies. [6]

FOXM1 expressed Nuclear, but can be detected in the cytoplasm upon inhibition of Raf/MEK/MAPK signaling. FoxM1 can also be targeted to the nucleolus by p19ARF. [7] FoxM1 is one of the most common genes overexpressed in solid tumors of prostate, lung, bladder, ovary, colon, liver, breast, kidney, stomach, and pancreas. [8] It is also aberrantly expressed in other cancers, such as basal cell carcinomas, glioblastomas, or acute myeloid leukemia [9] During the last years, significant progress has been made in targeting FoxM1 in cancer [10] Indeed, recent studies have already shown that targeting FoxM1 sensitizes different tumor cells to those DNA damaging treatments. [11], [12] Another interesting strategy is the possibility of combining FOXM1 inhibition with PARP inhibitors. PARP polymerases are required to repair single strand DNA breaks occurring during replication. When inhibited, 
unrepaired single-stranded DNA breaks result in stalled replication forks, which must be repaired by HR. Inhibiting HR, by means of FoxM1 suppression, may therefore sensitize these cells to PARP inhibitors. [10] Moreover, it is now becoming apparent that most conventional chemotherapies, which aim to induce extensive damage and apoptotic responses, are accompanied by a robust and concomitant induction of senescence. Interestingly, it is also now evident that senescent cells can be cleared in vivo through the innate immune response. [13] Therefore, combination of chemotherapy or IR with FOXM1 inhibition might improve the efficacy of those cancer treatments. Moreover, depending on the type of DNA lesion induced, FOXM1 inhibition might work at multiple levels, by blocking both DNA repair and checkpoint recovery processes, and promoting senescence. [14] FOXM1 gene is now known as a human proto-oncogene. [15] Abnormal up regulation of FOXM1 is involved in the oncogenesis of basal cell carcinoma, the most common human cancer worldwide. [16] FOXM1 up regulation was subsequently found in the majority of solid human cancers including liver, lung, prostate, cervix of uterus, colon, pancreas, brain and breast. [17]

FOXM1 promoted EMT in breast cancer by binding and activation of the promoter of SLUG gene [18] that FOXM1 promotes breast cancer metastases by activation of the TGF- $\beta$ pathway with through interaction SMAD 3 (this prevented E3 ubiquitin-protein ligase transcriptional intermediary factor $1 \gamma[$ TIF1 $\gamma]$ binding to SMAD3 and protected SMAD4 from ubiquitination) that leads to stabilization of the SMAD3/SMAD4 complex. [19] FOXM1 located in Nuclear, but can be detected in the cytoplasm upon inhibition of Raf/MEK/MAPK signaling. FoxM1 can also be targeted to the nucleolus by p19ARF [20]

\section{Material and Method}

The study samples included seventy four formalin-fixed, paraffin embedded tissue blocks which have been diagnosed as forty four cases of breast carcinoma and their lymph nodes obtained from the department of Pathology/ oncology hospital /Medical City during the period from January 2014 to January 2016, twenty cases diagnosis as benign breast tissue (fibro adenoma) obtained from ALSHAHEED GHAZI TEACHING HOSPITAL /medical city during January2014 to January 2016 and ten cases of normal breast tissue obtained from forensic medical center.

The diagnosis of each case (breast carcinoma, corresponding lymph node and fibro adenoma (was confirmed by examining the hematoxylin and eosin (H\&E) sections for histopathological reevaluation by two specialized pathologists.

The fresh breast tissue from cadaver were chosen as a sample of ten normal breast tissue. Demographic and clinical data provided by the surgeon were obtained from the surgical pathology reports available with the tissue specimens, including patient's age, sex, clinical presentation, site of the tumor, tumor size, histological subtype, lymph node involvement, grading, and staging. Then we stain for immunohistochemistry marker FOXM1, Positive controls For FOXM1 monoclonal antibody one tissue block of gall bladder was used. Negative control used for indicating the properness of the staining techniques, as positive control; one negative control was used for each set of test run.

\subsection{Principles of the Test}

Immunohistochemistry (IHC) enzyme labeled technique is a two-step indirect process, where the enzyme (peroxidase) is conjugated to a secondary reagent (link $\mathrm{Ab}$ ), providing an additional step for amplification of the antigen-antibody binding event. Biotin-Streptavidine amplified (B-SA) system is one of the most common linkers used in this method. A specific primary $\mathrm{Ab}$ will react with its corresponding antigen in the tissue, and then the biotin-labeled secondary antibody will bind to that primary $\mathrm{Ab}$. When the conjugate (Streptavidine bounded enzyme) is added, the biotinylated $\mathrm{Ab}$ will form a complex with the enzyme-conjugates streptavidin, and by adding the chromogene substrate, a colorimetric reaction will happen at the antigen binding site. The type of the chromogenic substrate depends on the type of the enzyme used. Thus, DAB (3, 3" diaminobenzidine) substrate offers the greatest sensitivity in the horseradish peroxidase enzyme system as a colorimetric chromogene, and a brownish precipitate will form at the antigen binding site.

\subsection{Evaluation of Immunohistochemistry Staining}

brown nuclear and cytoplasmic reaction was regarded positive for FOXM1. Immunohistochemical scoring was implemented by two independent observers who were blinded to the clinical data. the staining intensity of cells was classified into four intensity scores: ${ }^{23}$

No staining, 0; light staining, 1; moderate staining, 2; dark staining, 3 .

The percentage of positively-stained cells was classified into five grades with percentage scores as follows: $10 \%$ staining, $0 ; 10-25 \%$ staining, $1 ; 25-50 \%$ staining, $2 ; 50-75$ staining, 3 ; and $>75 \%$ staining, 4 .

The staining positivity of FoxM1 was calculated using the following formula: Total score $=$ intensity score $\times$ percentage score.

Based on the total score of each tissue, results of Negative $\leq 3$, Weakly positive $>3$ to $\leq 6$ Strong positive $>6$

\section{Result}

In the present study, 74 of the total studied cases $(62.2 \%)$ were negative for FOXM1 expression, and 28 of the total cases $(37.8 \%)$ were positive in expressing FOXM1. 


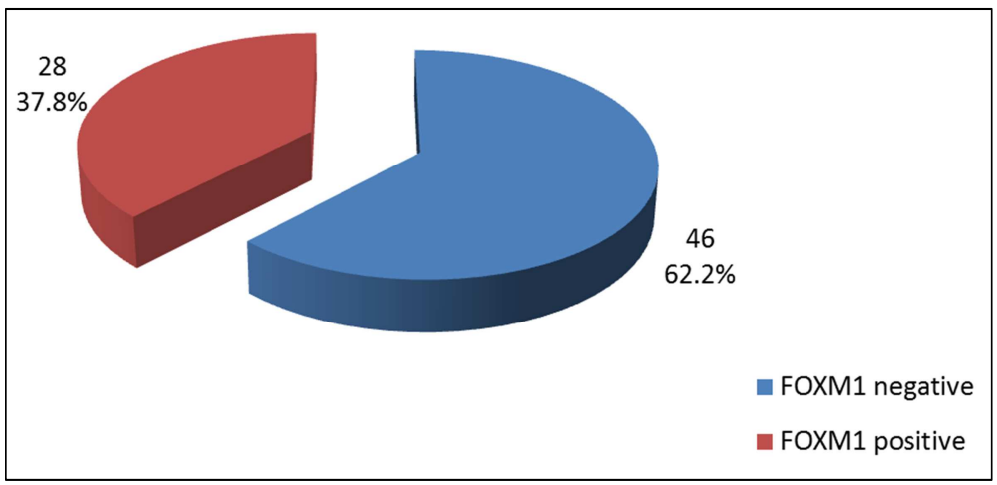

Figure 1. A pie chart showing FOXM1 immunohistochemistry expression in 74 cases (normal breast tissue, fibroadenoma, breast carcinoma).

Among malignant breast cancer 16 cases (36.4\%) showed no FOXM1 expression, while only 12 malignant cases (27.3\%) were strongly positive for FOXM1 expression. As shown in the figure below.

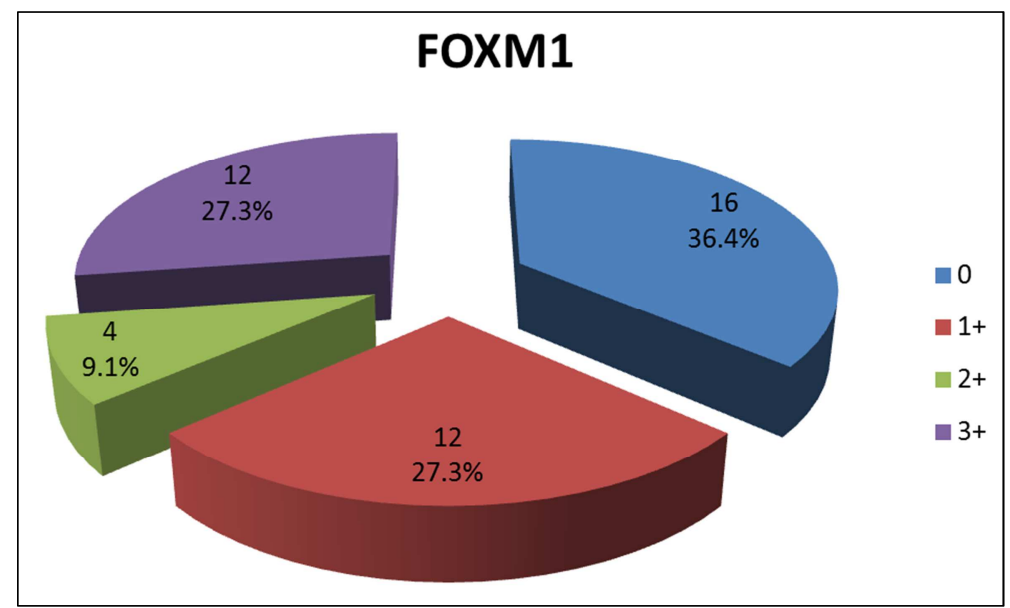

Figure 2. A pie chart showing expression of FOXM1 immunohistochemistry in malignant breast cases.

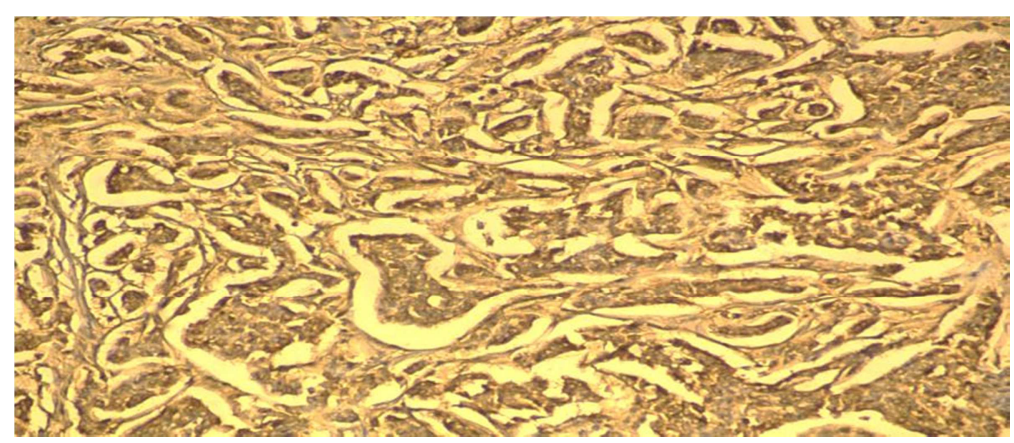

Figure 3. A slide view showing breast carcinoma stain strong positive for immunohistochemistry FOXM1marker.

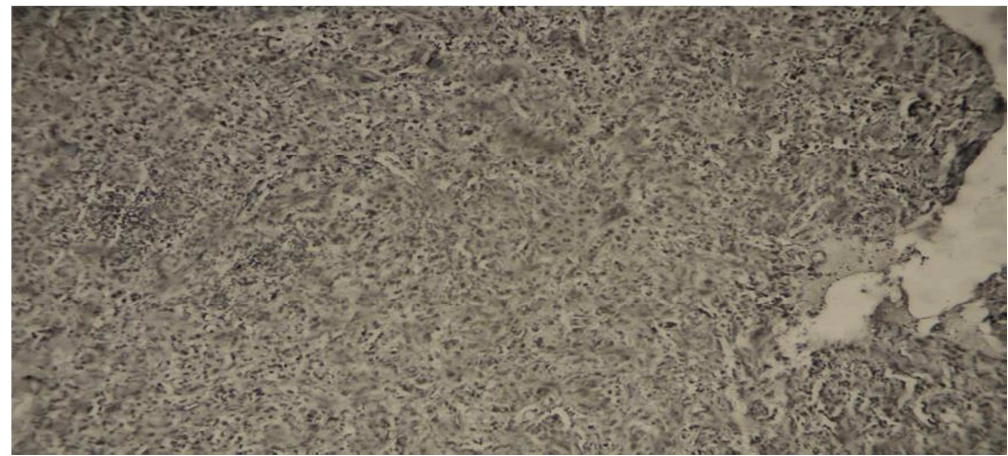

Figure 4. A slide picture showing breast carcinoma stain strong positive for immunohistochemistry FOXM1marker. 
Table 1. A table showing correlation between breast carcinoma grade and immunohistochemistry FOXM 1 expression.

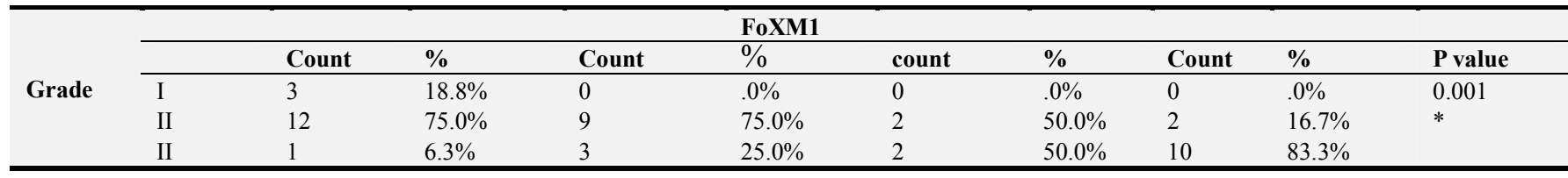

Table 2. A table showing correlation between breast carcinoma stage and immunohistochemistry FOXM 1 expression.

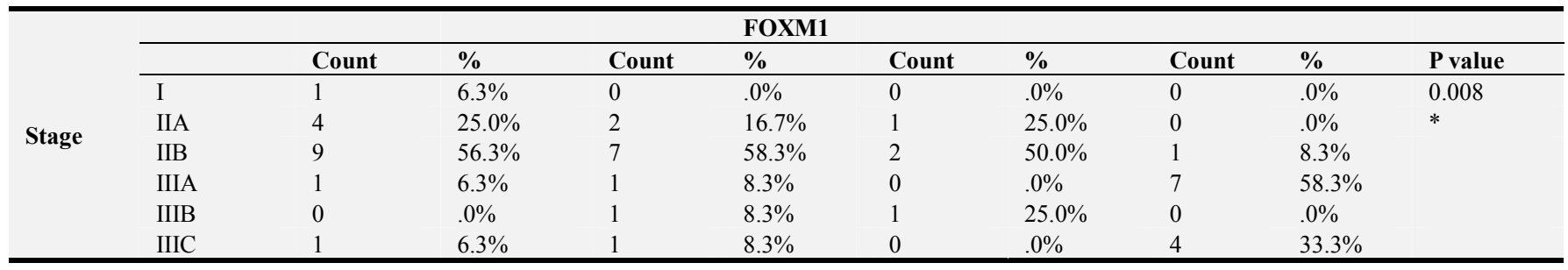

\section{Discussion}

In the present analysis FOXM1 is overexpressed in $37.8 \%$ of breast cancer samples, ten cases $(83.3 \%)$ of poorly differentiated breast carcinoma were showing strong expression of immunohistochemistry FOXM1 marker while the well differentiated breast carcinoma cases were shown weak expression of immunohistochemistry FOXM1 marker (all well differentiated cases was showing either no FOXM1 or weak expression ), J.-J. Lee et al. [21] has previously find the same result, CSCs are indeed enriched in high-grade breast cancers. That fact suggests that FOXM1 plays a critical role in angiogenesis, invasion, and metastasis. In addition to this, recent studies have placed FOXM 1 in DNA damage response and senescence pathways, two pathways relevant to tumor progression and the response to cancer therapies, In our ongoing study, we have noticed the higher expression levels of FOXM1 in more invasive breast cancer cells. This observation prompted us to investigate the physiologic/clinical relevance and the role of FOXM1 in the tumorigenesis of breast cancer.

In our study all fibro adenoma cases and normal breast tissue were showing no expression for FOXM1 immunohistochemistry marker, so we find normal no FOXM1 expression in normal tissue and no role of FOXM1 in benign breast tumor fibro adenoma

We also find significant correlation between FOXM1 immunohistochemistry and stage of breast carcinoma we find that all stage III carcinoma was shown strong expression of FOXM1 immunohistochemistry marker either score III or score II while stage I breast carcinoma cases were showing weak expression of FOXM1 immunohistochemistry marker score I, ZHANG et al [22] agree with our finding

\section{Conclusion}

FOXM1 marker is regarded as a predictive and a prognostic factor for breast carcinoma. In case with strong FOXM1 immunohistochemistry expression there will be an increase probability for the carcinoma to be more aggressive, high grade, larger size and an increase susceptibility for metastasis

\section{References}

[1] Susan G. Komen. What is breast cancer? Fact for life. 2016; $2 / 16$.

[2] Takahashi R, Takeshita F, Fujiwara T, Ono M, Ochiya T. Cancer stem cells in breast cancer. Cancers 2011; 3: 13111328.

[3] Clarke MF, Dick JE, Dirks PB, Eaves CJ, Jamieson CHM, Jones DL, Visvader J, Weissman IL, Wahl GM. Cancer StemCells-Perspectives on Current Status and Future Directions:AACR Workshop on Cancer Stem Cells. Cancer Res 2006; 66: 9339-9344.

[4] Chan SW, Lim CJ, Guo K, Ng CP, Lee I, Hunziker W, Zeng Q, Hong W. A role for TAZ in migration, invasion, and tumorigenesis of breast cancer cells. Cancer Res. Apr. 2008; 68 (8):2592-2598.

[5] Ye H, Kelly TF, Samadani U, Lim L, Rubio S, Overdier DG, Roebuck KA, Costa RH. "Hepatocyte nuclear factor 3/fork head homolog 11 is expressed in proliferating epithelial and mesenchymal cells of embryonic and adult tissues". Mol. Cell. Biol. 1997; 17 (3): 1626-41.

[6] Vincent Shen. "2010 Molecule of the Year". BioTechniques.

[7] Laoukili J, Kooistra MR, Brás A, Kauw J, Kerkhoven RM, Morrison A, Clevers H, Medema RH. FoxM1 is required for execution of the mitotic programme and chromosome stability. Nat. Cell Biol. 2005; 7 (2): 126-36.

[8] Pilarsky, C., Wenzig, M., Specht, T., Saeger, H. D., and Grutzmann, R. Identification and validation of commonly overexpressed genes in solid tumors by comparison of microarray data. Neoplasia. 2004; 6, 744-750.

[9] Nakamura, S., Hirano, I., Okinaka, K., Takemura, T., Yokota, D., Ono, T., et al. The FOXM1 transcriptional factor promotes the proliferation of leukemia cells through modulation of cell cycle progression in acute myeloid leukemia. Carcinogenesis. 2010; 31, 2012-2021.

[10] Kwok, J. M., Peck, B., Monteiro, L. J., Schwenen, H. D., Millour, J., Coombes, R. C., et al. FOXM1 confers acquired cisplatin resistance in breast cancer cells. Mol. Cancer Res. 2010; 8, 24-34. 
[11] Zhang, N., Wu, X., Yang, L., Xiao, F., Zhang, H., Zhou, A., et al. FoxM1 inhibition sensitizes resistant glioblastoma cells to temozolomide by downregulating the expression of DNArepair gene Rad51. Clin. Cancer Res. 2010a; 18, 5961-5971.

[12] Halasi, M., and Gartel, A. L. Suppression of FOXM1 sensitizes human cancer cells to cell death induced by DNAdamage. PLoS ONE. 2012a; 7:e31761.

[13] Xue, W., Zender, L., Miething, C., Dickins, R. A., Hernando, E., Krizhanovsky, V., et al. Senescence and tumour clearance is triggered by p53 restoration in murine liver carcinomas. Nature. 2007;-445, 656-660.

[14] Radhakrishnan, S. K., Bhat, U. G., Hughes, D. E., Wang, I. C., Costa, R. H., and Gartel, A. L. Identification of a chemical inhibitor of the oncogenic transcription factor forkhead box M1. Cancer Res. 2006; 66, 9731-9735.

[15] Myatt, S. S., and Lam, E. W. The emerging roles of forkhead box (Fox) proteins in cancer. Nat. Rev. Cancer. 2007; 7, 847859 .

[16] Pilarsky, C., Wenzig, M., Specht, T., Saeger, H. D., and Grutzmann, R. Identification and validation of commonly overexpressed genes in solid tumors by comparison of microarray data. Neoplasia. 2004; 6, 744-750.

[17] Wonsey DR, Follettie MT. "Loss of the forkhead transcription factor FoxM1 causes centrosome amplification and mitotic catastrophe". Cancer Res. 2005; 65 (12): 5181-9.

[18] C. Yang, H. Chen, G. Tan et al., "FOXM1 promotes the epithelial to mesenchymal transition by stimulating the transcription of Slug in human breast cancer," Cancer Letters. 2013; vol. 340, no. 1, pp. 104-112.

[19] J. Xue, X. Lin, W.-T. Chiu et al., "Sustained activation of SMAD3/SMAD4 by FOXM1 promotes TGF- $\beta$-dependent cancer metastasis," The Journal of Clinical Investigation. 2014; vol. 124, no. 2, pp. 564-579.

[20] J. Laoukili, M. Alvarez Fernandez, R. H Medema." FOXM1 (forkhead box M1)", Atlas of Genetics and Cytogenetics in Oncology and Haematology, 2008-2.

[21] Jeong-Ju Lee, Hee Jin Lee, Byung-Ho Son, Sung-Bae Kim, Jin-Hee Ahn, Seung Do Ahn, Eun Yoon Cho and Gyungyub Gong. Expression of FOXM1 and related proteins in breast cancer molecular subtypes. INTERNATIONAL JOURNAL OF EXPERIMENTAL PATHOLOGY. 2016; 97, 170-177.

[22] Jing zhang, kundong zhang, lisheng zhou, weidong wu, tao jun cao, kejian huang, zhengjun qiu and chen huang. Expression and potential correlation among Forkhead box protein M1, Caveolin-1 and E-cadherin in colorectal cancer. ONCOLOGY LETTERS. 2016; 12; 2381-2388. 